\title{
Blow-Up in a Slow Diffusive $p$-Laplace Equation with the Neumann Boundary Conditions
}

\author{
Chengyuan Qu ${ }^{1,2}$ and Bo Liang ${ }^{3}$ \\ ${ }^{1}$ Department of Mathematics, Dalian Nationalities University, Dalian 116600, China \\ ${ }^{2}$ School of Science, East China Institute of Technology, Nanchang 330013, China \\ ${ }^{3}$ School of Science, Dalian Jiaotong University, Dalian 116028, China
}

Correspondence should be addressed to Chengyuan Qu; mathqcy@163.com

Received 4 April 2013; Accepted 4 June 2013

Academic Editor: Daniel C. Biles

Copyright (C) 2013 C. Qu and B. Liang. This is an open access article distributed under the Creative Commons Attribution License, which permits unrestricted use, distribution, and reproduction in any medium, provided the original work is properly cited.

We study a slow diffusive $p$-Laplace equation in a bounded domain with the Neumann boundary conditions. A natural energy is associated to the equation. It is shown that the solution blows up in finite time with the nonpositive initial energy, based on an energy technique. Furthermore, under some assumptions of initial data, we prove that the solutions with bounded initial energy also blow up.

\section{Introduction}

In this paper, we consider a slow diffusive $p$-Laplace equation:

$$
\begin{gathered}
u_{t}-\operatorname{div}\left(|\nabla u|^{p-2} \nabla u\right)=|u|^{q-1} u-f_{\Omega}|u|^{q-1} u d x, \\
(x, t) \in \Omega \times(0, T), \\
\frac{\partial u}{\partial n}=0, \quad(x, t) \in \partial \Omega \times(0, T), \\
u(x, 0)=u_{0}(x), \quad x \in \Omega
\end{gathered}
$$

with $f_{\Omega} u_{0} d x=0$, where $\Omega$ is a bounded smooth domain $\Omega \subset$ $\mathbb{R}^{N}, p>2, q>p-1$, and $u_{0} \in L^{\infty}(\Omega) \cap W^{1, p}(\Omega), u_{0} \neq \equiv 0$, and denote $f_{\Omega} f d x=(1 /|\Omega|) \int_{\Omega} f d x$. It is easy to check that $\int_{\Omega} u d x=0$; that is, the mass of $u$ is conserved.

The problem (1) with $p=2$ can be used to model phenomena in population dynamics and biological sciences where the total mass of a chemical or an organism is conserved $[1,2]$. If $p>2$, the problem (1) is the degenerate parabolic equation and appears to be relevant in the theory of non-Newtonian fluids (see [3]). Here, we are mainly interested in the case $p>2$, namely, the degenerate one.
When $p=2$, (1) becomes the heat equation which has been deeply studied in $[4,5]$. When $1<p<2,(1)$ is singular, which can be handled similar to that of [6].

As an important feature of many evolutionary equations, the properties of blow-up solution have been the subject of intensive study during the last decades. Among those investigations in this area, it was Fujita [7] who first established the so-called theory of critical blow-up exponents for the heat equation with reaction sources in 1966, which can be, of course, regarded as the elegant description for either blowup or global existence of solutions. From then on, there has been increasing interest in the study of critical Fujita exponents for different kinds of evolutionary equations; see $[8,9]$ for a survey of the literature. In recent years, special attention has been paid to the blow-up property to nonlinear degenerate or singular diffusion equations with different nonlinear sources, including the inner sources, boundary flux, or multiple sources; see, for example, $[3,10,11]$.

In some situations, we have to deal with changing sign solutions. For instance, the changing sign solutions were considered in [1] for the nonlocal and quadratic equation

$$
u_{t}=\Delta u+u^{2}-f_{\Omega} u^{2} d x
$$


with the Neumann boundary condition. The study in [5] for

$$
u_{t}=\Delta u+|u|^{p}-f_{\Omega}|u|^{p} d x,
$$

a natural generalization of (2), proposed with $1<p \leq 2$ a global existence result (for small initial data) and a new blow-up criterion (based on the partial maximum principle and a Gamma-convergence argument). The authors also conjectured that the solutions blow up when $p>2$, which was then proved with a positive answer [4]. The changing sign solutions to the reaction-diffusion equation

$$
u_{t}=\Delta u+f(u, k(t))
$$

were discussed in [2], with such as $f(u, k(t))=|u|^{p-1} u-k(t)$. The blow-up of solutions was obtained even under the source with $f_{\Omega} f d x=0$. The semilinear parabolic equation [12]

$$
u_{t}=\Delta u+|u|^{p-1} u-f_{\Omega}|u|^{p-1} u d x
$$

with a homogeneous Neumann's boundary condition is studied. A blow-up result for the changing sign solution with positive initial energy is established. In [6], a fast diffusive $p$ Laplace equation with the nonlocal source

$$
\begin{gathered}
u_{t}-\operatorname{div}\left(|\nabla u|^{p-2} \nabla u\right)=|u|^{q}-f_{\Omega}|u|^{q} d x, \\
(x, t) \in \Omega \times(0, T), \\
\frac{\partial u}{\partial n}=0, \quad(x, t) \in \partial \Omega \times(0, T), \\
u(x, 0)=u_{0}(x), \quad x \in \Omega,
\end{gathered}
$$

was considered. The authors showed that a critical blowup criterion was determined for the changing sign weak solutions, depending on the size of $q$ and the sign of the natural energy associated. The relationship between the finite time blow-up and the nonpositivity of initial energy was discussed, based on an energy technique.

Notice that (1) is degenerate if $p>2$ at points where $\nabla u=$ 0 ; therefore, there is no classical solution in general. For this, a weak solution for problem (1) is defined as follows.

Definition 1. A function $u \in L^{\infty}(\Omega \times(0, T)) \cap L^{p}(0, T$, $\left.W^{1, p}(\Omega)\right)$ with $u_{t} \in L^{2}(\Omega \times(0, T))$ is called a weak solution of (1) if

$$
\begin{gathered}
\int_{0}^{t} \int_{\Omega}\left[u \frac{\partial \varphi}{\partial s}-|\nabla u|^{p-2} \nabla u \cdot \nabla \varphi+\left(|u|^{q}-f_{\Omega}|u|^{q}\right) \varphi\right] d x d s \\
=\int_{\Omega} u(x, t) \varphi(x, t) d x-\int_{\Omega} u_{0}(x) \varphi(x, 0) d x
\end{gathered}
$$

holds for all $\varphi \in C^{1}(\bar{\Omega} \times[0, T])$.

The local existence of the weak solutions can be obtained via the standard procedure of regularized approximations
[10]. Throughout the paper, we always assume that the weak solution is appropriately smooth for convenience of arguments, instead of considering the corresponding regularized problems.

This paper is organized as follows. In Section 2, we show that the solutions to (1) blow up with nonpositive initial energy. In Section 3, under some assumptions of initial data, we prove that the solutions with bounded initial energy also blow up in finite time.

\section{Nonpositive Initial Energy Case}

The technique used here is the same as in [4]; define the energy functional by

$$
E(t)=\frac{1}{p} \int_{\Omega}|\nabla u|^{p} d x-\frac{1}{q+1} \int_{\Omega}|u|^{q+1} d x .
$$

and denote

$$
M(t)=\frac{1}{2} \int_{\Omega} u^{2}(x, t) d x, \quad H(t)=\int_{0}^{t} M(s) d s .
$$

Theorem 2. Assume that $p>2, q>p-1$, and $u_{0} \in L^{\infty}(\Omega) \cap$ $W^{1, p}(\Omega), u_{0} \neq \equiv$, and let the initial energy

$$
E(0)=\frac{1}{p} \int_{\Omega}\left|\nabla u_{0}\right|^{p} d x-\frac{1}{q+1} \int_{\Omega}\left|u_{0}\right|^{q+1} d x
$$

be nonpositive. Then, there exists $T_{0}$ with $0<T_{0}<\infty$, such that

$$
\lim _{t \rightarrow T_{0}} M(t)=+\infty .
$$

We need three lemmas for the functionals $E(t), M(t)$, and $H(t)$, respectively.

Lemma 3. The energy $E(t)$ is a nonincreasing function and

$$
E(t)=E(0)-\int_{0}^{t} \int_{\Omega}\left(u_{t}\right)^{2} d x d s .
$$

Proof. A direct computation using (1) and by parts yields

$$
\begin{aligned}
\frac{d}{d t} E(t) & =\int_{\Omega}\left(|\nabla u|^{p-2} \nabla u \cdot \nabla u_{t}-|u|^{q-1} u u_{t}\right) d x \\
& =\int_{\Omega}\left(-\operatorname{div}\left(|\nabla u|^{p-2} \nabla u\right)-|u|^{q-1} u\right) u_{t} d x \\
& =\int_{\Omega}\left(-u_{t}-f_{\Omega}|u|^{q-1} u d x\right) u_{t} d x \\
& =-\int_{\Omega}\left(u_{t}\right)^{2} d x .
\end{aligned}
$$

Integrate from 0 to $t$ to get (12).

Lemma 4. Assume that $p>2, q>p-1$, and $E(0) \leq 0$. Then, $M(t)$ satisfies the following inequality:

$$
M^{\prime}(t) \geq(q+1) \int_{0}^{t} \int_{\Omega}\left(u_{t}\right)^{2} d x d s .
$$


Proof. An easy computation using (1) and the fact $\int_{\Omega} u d x=0$ and by parts shows that

$$
\begin{aligned}
M^{\prime}(t) & =\int_{\Omega} u u_{t} d x \\
& =\int_{\Omega} u\left(\operatorname{div}\left(|\nabla u|^{p-2} \nabla u\right)+|u|^{q-1} u-f_{\Omega}|u|^{q-1} u d x\right) \\
& =-\int_{\Omega}|\nabla u|^{p} d x+\int_{\Omega}|u|^{q+1} d x \\
& =-(q+1) E(t)+\frac{q+1-p}{p} \int_{\Omega}|\nabla u|^{p} d x .
\end{aligned}
$$

The last equality implies

$$
\begin{aligned}
M^{\prime}(t) & \geq-(q+1) E(t) \\
& =-(q+1) E(0)+(q+1) \int_{0}^{t} \int_{\Omega}\left(u_{t}\right)^{2} d x d s \\
& \geq(q+1) \int_{0}^{t} \int_{\Omega}\left(u_{t}\right)^{2} d x d s,
\end{aligned}
$$

because of (12) of Lemma 3 and the assumption $E(0) \leq 0$.

Lemma 5. Assume that $p>2, q>p-1$, and $E(0) \leq 0$. Then, $H(t)$ satisfies

$$
\frac{q+1}{2}\left(H^{\prime}(t)-H^{\prime}(0)\right)^{2} \leq H(t) H^{\prime \prime}(t) .
$$

Proof. Note the definition of $M(t)$ and $H(t)$, and a simple calculation shows that

$$
\begin{aligned}
H^{\prime}(t) & -H^{\prime}(0) \\
& =M(t)-M(0) \\
& =\int_{0}^{t} M^{\prime}(s) d s=\int_{0}^{t} \int_{\Omega} u u_{t} d x d s \\
& \leq\left(\int_{0}^{t} \int_{\Omega} u^{2} d x d s\right)^{1 / 2}\left(\int_{0}^{t} \int_{\Omega}\left(u_{t}\right)^{2} d x d s\right)^{1 / 2} \\
& \leq\left(\frac{2}{q+1}\right)^{1 / 2}(H(t))^{1 / 2}\left(M^{\prime}(t)\right)^{1 / 2} \\
& =\left(\frac{2}{q+1}\right)^{1 / 2}(H(t))^{1 / 2}\left(H^{\prime \prime}(t)\right)^{1 / 2} .
\end{aligned}
$$

Furthermore,

$$
\begin{aligned}
H^{\prime}(t)-H^{\prime}(0) & =\int_{0}^{t} M^{\prime}(s) d s \\
& \geq(q+1) t \int_{0}^{t} \int_{\Omega}\left(u_{t}\right)^{2} d x d s \geq 0 .
\end{aligned}
$$

Therefore,

$$
\frac{q+1}{2}\left(H^{\prime}(t)-H^{\prime}(0)\right)^{2} \leq H(t) H^{\prime \prime}(t) .
$$

Proof of Theorem 2. Assume for contradiction that the solution $u$ exists for all $t>0$. We claim that

$$
\int_{0}^{t_{0}} \int_{\Omega}\left(u_{t}\right)^{2} d x d s>0
$$

for any $t_{0}>0$. Otherwise, there exists $t_{0}>0$ such that

$$
\int_{0}^{t_{0}} \int_{\Omega}\left(u_{t}\right)^{2} d x d s=0
$$

and hence $u_{t}=0$ for a.e. $(x, t) \in \Omega \times\left(0, t_{0}\right]$. Therefore, noticing $E(t) \leq 0$ by Lemma 3, we have from (15) that

$$
\int_{\Omega}|\nabla u|^{p} d x=0
$$

for a.e. $t \in\left(0, t_{0}\right]$. Using the Poincaré inequality with $\int_{\Omega} u d x=0$, we have $u=0$ for a.e. $(x, t) \in \Omega \times\left(0, t_{0}\right]$. This contradicts $u_{0} \not \equiv 0$.

Integrating (14) from $t_{0}$ to $t$, we have

$$
M(t) \geq M\left(t_{0}\right)+(q+1) \int_{t_{0}}^{t} \int_{0}^{\tau} \int_{\Omega}\left(u_{t}\right)^{2} d x d s d \tau,
$$

which implies that

$$
\lim _{t \rightarrow \infty} H^{\prime}(t)=\lim _{t \rightarrow \infty} M(t)=+\infty
$$

Thus, there exists $t^{*} \geq t_{0}$ such that for all $t \geq t^{*}$

$$
\frac{3 q+5}{4}\left(H^{\prime}(t)\right)^{2} \leq(q+1)\left[H^{\prime}(t)-H^{\prime}(0)\right]^{2} .
$$

Thus, combining (17), we further have

$$
\frac{3 q+5}{4}\left(H^{\prime}(t)\right)^{2} \leq 2 H(t) H^{\prime \prime}(t)
$$

for all $t \geq t^{*}$. Now, we consider the function $G(t)=$ $(H(t))^{-((q-1) / 4)}$. Combining with the above inequality and a simple calculation shows that

$$
\begin{aligned}
G^{\prime \prime}(t)= & \frac{q-1}{4}(H(t))^{(-q-7) / 4} \\
& \times\left(\frac{q+3}{4}\left(H^{\prime}(t)\right)^{2}-H(t) H^{\prime \prime}(t)\right) \\
\leq & -\frac{(q-1)^{2}}{32}(H(t))^{(-q-7) / 4}\left(H^{\prime}(t)\right)^{2} \leq 0
\end{aligned}
$$

for all $t \geq t^{*}$. However, since

$$
\lim _{t \rightarrow \infty} H(t)=\lim _{t \rightarrow \infty} M(t)=\infty,
$$

we also have

$$
\lim _{t \rightarrow \infty} G(t)=0
$$

which is a contradiction. 


\section{Bounded Initial Energy Case}

Define

$$
W(\Omega)=\left\{u \in W^{1, p}(\Omega) \mid \int_{\Omega} u d x=0\right\}
$$

with the norm $\|u\|=\left(\int_{\Omega}|\nabla u|^{p} d x\right)^{1 / p}$. Let $B$ be the optimal constant of the embedding inequality

$$
\|u\|_{q+1} \leq B\|\nabla u\|_{p}
$$

where $p-1<q \leq\left(N p /(N-p)_{+}\right)-1$. Set

$$
\begin{gathered}
\alpha_{1}=B^{-(q+1) /(q-p+1)}, \\
E_{1}=\left(\frac{1}{p}-\frac{1}{q+1}\right) B^{-p(q+1) /(q-p+1)}>0 .
\end{gathered}
$$

Theorem 6. Assume that $p>2, p-1<q \leq\left(N p /(N-p)_{+}\right)-1$. Let the initial data $u_{0}$ satisfying $E(0) \leq E_{1}$ and $\left\|\nabla u_{0}\right\|_{p}>\alpha_{1}$. Then, there exists $T_{1}$ with $0<T_{1}<\infty$, such that

$$
\lim _{t \rightarrow T_{1}} M(t)=+\infty
$$

First, we prove the following two Lemmas, similar to the idea in [13].

Lemma 7. Assume that $u$ is a solution of the system (1). If $E(0)<E_{1}$ and $\left\|\nabla u_{0}\right\|_{p}>\alpha_{1}$. Then, there exists a positive constant $\alpha_{2}>\alpha_{1}$, such that

$$
\begin{gathered}
\|\nabla u\|_{p} \geq \alpha_{2}, \quad \text { for any } t \geq 0, \\
\|u\|_{q+1} \geq B \alpha_{2}, \quad \text { for any } t \geq 0 .
\end{gathered}
$$

Proof. Let $\|\nabla u\|_{p}=\alpha$ and by (32), we have

$$
\begin{aligned}
E(t) & =\frac{1}{p} \int_{\Omega}|\nabla u|^{p} d x-\frac{1}{q+1} \int_{\Omega}|u|^{q+1} d x \\
& \geq \frac{1}{p}\|\nabla u\|_{p}^{p}-\frac{1}{q+1} B^{q+1}\|\nabla u\|_{p}^{q+1} \\
& =\frac{1}{p} \alpha^{p}-\frac{1}{q+1} B^{q+1} \alpha^{q+1} .
\end{aligned}
$$

For convenience, we define

$$
g(\alpha)=\frac{1}{p} \alpha^{p}-\frac{1}{q+1} B^{q+1} \alpha^{q+1} .
$$

It is easy to find that $g$ increases if $0<\alpha<\alpha_{1}$ and decreases if $\alpha>\alpha_{1}$. Moreover, $g(\alpha) \rightarrow-\infty$ as $\alpha \rightarrow \infty$ and $g\left(\alpha_{1}\right)=E_{1}$. Due to $E(0)<E_{1}$, there exists $\alpha_{2}>\alpha_{1}$ such that $g\left(\alpha_{2}\right)=$ $E(0)$. Let $\left\|\nabla u_{0}\right\|_{p}=\alpha_{0}$; thus $\alpha_{0}>\alpha_{1}$. Then by (37) and (38), we have $g\left(\alpha_{0}\right) \leq E(0)=g\left(\alpha_{2}\right)$, which implies that $\alpha_{0} \geq \alpha_{2}$. For contradiction to establish (35), we assume that there exists $t_{0}>0$ such that

$$
\alpha_{1}<\left\|\nabla u\left(\cdot, t_{0}\right)\right\|_{p}<\alpha_{2} .
$$

It follows from (37) and (38) that

$$
E\left(t_{0}\right) \geq g\left(\left\|\nabla u\left(\cdot, t_{0}\right)\right\|_{p}\right)>g\left(\alpha_{2}\right)=E(0),
$$

which is in contradiction with Lemma 3. Hence, (35) is established.

Next to prove (36),

$$
E(t)=\frac{1}{p} \int_{\Omega}|\nabla u|^{p} d x-\frac{1}{q+1} \int_{\Omega}|u|^{q+1} d x \leq E(0),
$$

which implies that

$$
\frac{1}{q+1} \int_{\Omega}|u|^{q+1} d x \geq \frac{1}{p} \int_{\Omega}|\nabla u|^{p} d x-E(0) \geq \frac{1}{p} \alpha_{2}^{p}-g\left(\alpha_{2}\right) .
$$

Therefore, (36) is concluded.

Define

$$
F(t)=E_{1}-E(t), \quad \text { for any } t \geq 0 .
$$

Then, we have the following.

Lemma 8. Assume that $u$ is a solution of the system (1). If $E(0)<E_{1}$ and $\left\|\nabla u_{0}\right\|_{p}>\alpha_{1}$. Then for all $t \geq 0$,

$$
0<F(0) \leq F(t) \leq \frac{1}{q+1} \int_{\Omega}|u|^{q+1} d x
$$

Proof. By Lemma 3, we know that $F^{\prime}(t) \geq 0$. Thus,

$$
F(t) \geq F(0)=E_{1}-E(0)>0 .
$$

According to (35) of Lemma 7, a simple computation shows that

$$
\begin{aligned}
F(t) & =E_{1}-\frac{1}{p} \int_{\Omega}|\nabla u|^{p} d x+\frac{1}{q+1} \int_{\Omega}|u|^{q+1} d x \\
& \leq E_{1}-\frac{1}{p} B^{-p(q+1) /(q-p+1)}+\frac{1}{q+1} \int_{\Omega}|u|^{q+1} d x \\
& =-\frac{1}{q+1} B^{-p(q+1) /(q-p+1)}+\frac{1}{q+1} \int_{\Omega}|u|^{q+1} d x \\
& \leq \frac{1}{q+1} \int_{\Omega}|u|^{q+1} d x,
\end{aligned}
$$

which guarantees the conclusion of the lemma.

At the end, let us finish the proof of Theorem 6 .

Proof of Theorem 6. According to (15), we have

$$
\begin{aligned}
M^{\prime}(t) & =-\int_{\Omega}|\nabla u|^{p} d x+\int_{\Omega}|u|^{q+1} d x \\
& =\int_{\Omega}|u|^{q+1} d x-p E(t)-\frac{p}{q+1} \int_{\Omega}|u|^{q+1} d x \\
& =\frac{q+1-p}{q+1} \int_{\Omega}|u|^{q+1} d x-p E_{1}+p F(t) .
\end{aligned}
$$


By using (33) and (36), we obtain

$$
\begin{aligned}
p E_{1} & =\left(1-\frac{p}{q+1}\right) B^{-p(q+1) /(q+1-p)} \\
& =\frac{\alpha_{1}^{q+1}}{\alpha_{2}^{q+1}} \frac{q+1-p}{q+1} B^{q+1} \alpha_{2}^{q+1} \\
& \leq \frac{\alpha_{1}^{q+1}}{\alpha_{2}^{q+1}} \frac{q-p+1}{q+1} \int_{\Omega}|u|^{q+1} d x .
\end{aligned}
$$

Combining (47) and (48), we get

$$
\begin{aligned}
M^{\prime}(t) & \geq\left(1-\frac{\alpha_{1}^{q+1}}{\alpha_{2}^{q+1}}\right) \frac{q+1-p}{q+1} \int_{\Omega}|u|^{q+1} d x+p F(t) \\
& \geq\left(1-\frac{\alpha_{1}^{q+1}}{\alpha_{2}^{q+1}}\right) \frac{q+1-p}{q+1}|\Omega|^{(1-q) / 2} M^{(q+1) / 2} .
\end{aligned}
$$

Since $q>p-1>1, M(t)$ blows up at a finite time. The proof of Theorem 6 is complete.

Remark 9 (behavior of the energy $E(t)$ ). Similar to Theorem 1.3 of [5], it is easy to be proved. Let $p>2, p-1<q \leq$ $\left(\mathrm{Np} /(\mathrm{N}-\mathrm{p})_{+}\right)-1$, and let $u$ be a weak solution of (1). If there exists a constant $C_{0}>0$ and a time $T_{0}^{\prime}>0$, such that the solution $u$ exists on $\left[0, T_{0}^{\prime}\right)$ and satisfies $E(t) \geq-C_{0}$ on $\left[0, T_{0}^{\prime}\right)$, then $F(t)$ is bounded on $\left[0, T_{0}^{\prime}\right)$. Thus, the above result and Theorem 6 reveal that even though the initial energy could be chosen as positive, the energy $E(t)$ needs to become negative at a certain time and then goes to $-\infty$. Otherwise, $E(t)$ has a lower bound on $[0,+\infty)$; thus $F(t)$ is bounded on $[0,+\infty)$. It is in contradiction with Theorem 6 .

\section{Acknowledgments}

This study was supported by the National Natural Science Foundation of China (Grants nos. 11226179, 11201045) and the Doctor Startup Fund of Dalian Nationalities University (Grant no. 0701-110030).

\section{References}

[1] C. Budd, B. Dold, and A. Stuart, "Blowup in a partial differential equation with conserved first integral," SIAM Journal on Applied Mathematics, vol. 53, no. 3, pp. 718-742, 1993.

[2] B. Hu and H.-M. Yin, "Semilinear parabolic equations with prescribed energy," Rendiconti del Circolo Matematico di Palermo. Serie II, vol. 44, no. 3, pp. 479-505, 1995.

[3] N. D. Alikakos and L. C. Evans, "Continuity of the gradient for weak solutions of a degenerate parabolic equation," Journal de Mathématiques Pures et Appliquées. Neuvième Série, vol. 62, no. 3, pp. 253-268, 1983.

[4] M. Jazar and R. Kiwan, "Blow-up of a non-local semilinear parabolic equation with Neumann boundary conditions," Annales de l'Institut Henri Poincaré. Analyse Non Linéaire, vol. 25, no. 2, pp. 215-218, 2008.

[5] A. El Soufi, M. Jazar, and R. Monneau, "A gamma-convergence argument for the blow-up of a non-local semilinear parabolic equation with Neumann boundary conditions," Annales de l'Institut Henri Poincaré. Analyse Non Linéaire, vol. 24, no. 1, pp. 17-39, 2007.

[6] C. Y. Qu, X. L. Bai, and S. N. Zheng, "Blow-up and extinction in a nonlocal $p$-Laplace equation with Neumann boundary conditions," Annales de l'Institut Henri Poincare (C) Non Linear Analysis, vol. 25, no. 2, pp. 215-218, 2008.

[7] H. Fujita, "On the blowing up of solutions of the Cauchy problem for $u_{t}=\Delta u+u^{1+\alpha}$," Journal of the Faculty of Science. University of Tokyo. Section IA. Mathematics, vol. 13, pp. 109-124, 1966.

[8] K. Deng and H. A. Levine, "The role of critical exponents in blow-up theorems: the sequel," Journal of Mathematical Analysis and Applications, vol. 243, no. 1, pp. 85-126, 2000.

[9] Y. X. Li and C. H. Xie, "Blow-up for $p$-Laplace parabolic equations," Electronic Journal of Differential Equations, vol. 2003, pp. 1-12, 2005.

[10] J. N. Zhao, "Existence and nonexistence of solutions for $u_{t}=$ $\operatorname{div}\left(|\nabla u|^{p-2} \nabla u\right)+f(\nabla u, u, x, t)$, , Journal of Mathematical Analysis and Applications, vol. 172, no. 1, pp. 130-146, 1993.

[11] S. Zheng, X. Song, and Z. Jiang, "Critical Fujita exponents for degenerate parabolic equations coupled via nonlinear boundary flux," Journal of Mathematical Analysis and Applications, vol. 298, no. 1, pp. 308-324, 2004.

[12] W. Gao and Y. Han, "Blow-up of a nonlocal semilinear parabolic equation with positive initial energy," Applied Mathematics Letters, vol. 24, no. 5, pp. 784-788, 2011.

[13] E. Vitillaro, "Global nonexistence theorems for a class of evolution equations with dissipation," Archive for Rational Mechanics and Analysis, vol. 149, no. 2, pp. 155-182, 1999. 


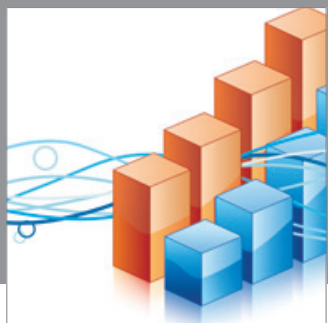

Advances in

Operations Research

mansans

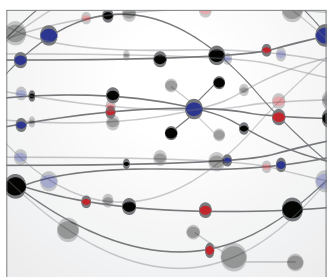

The Scientific World Journal
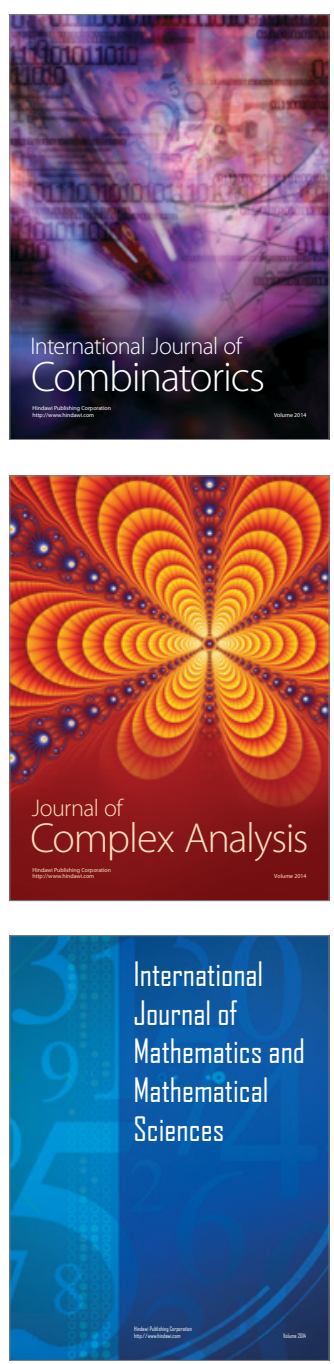
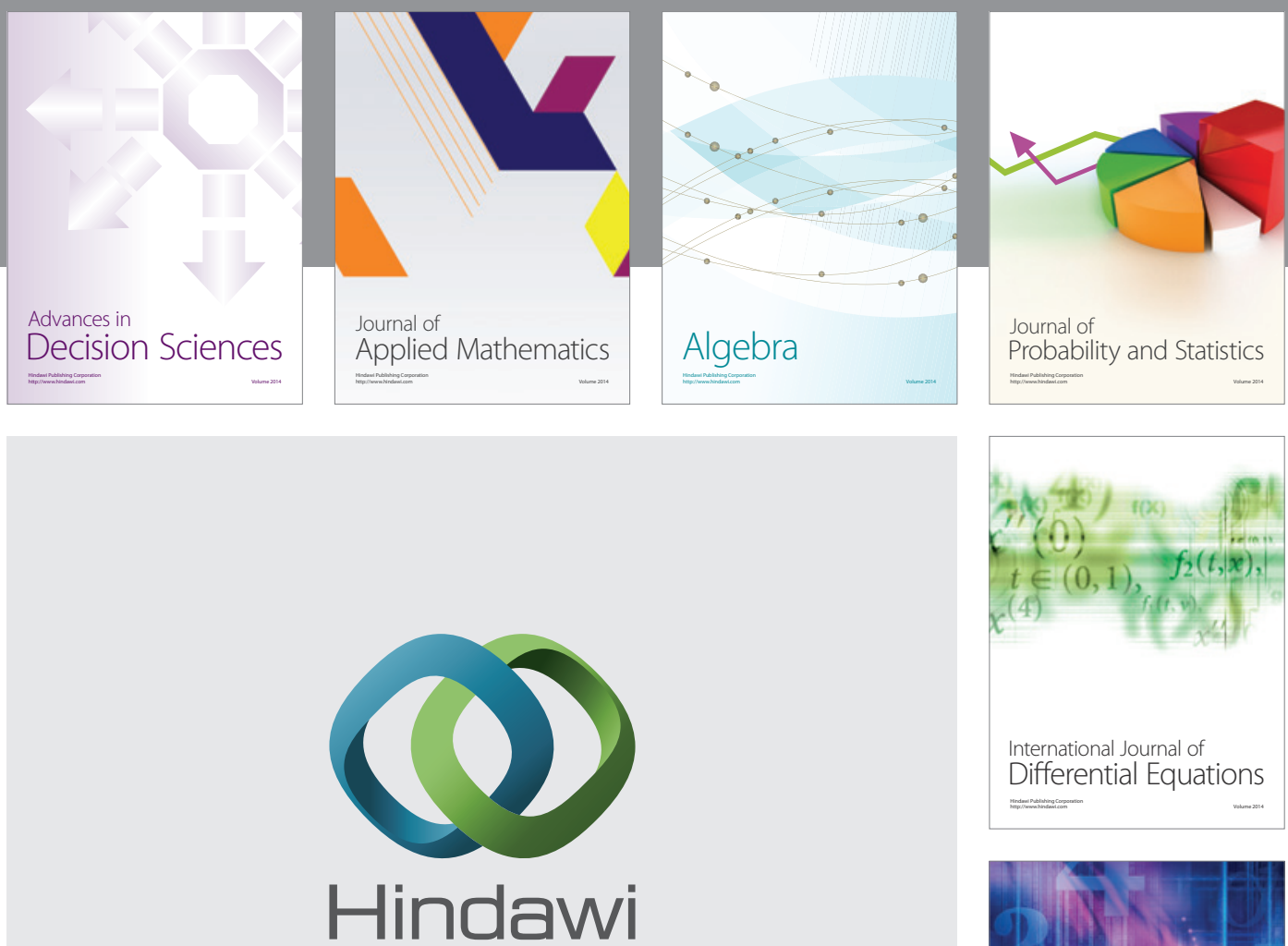

Submit your manuscripts at http://www.hindawi.com
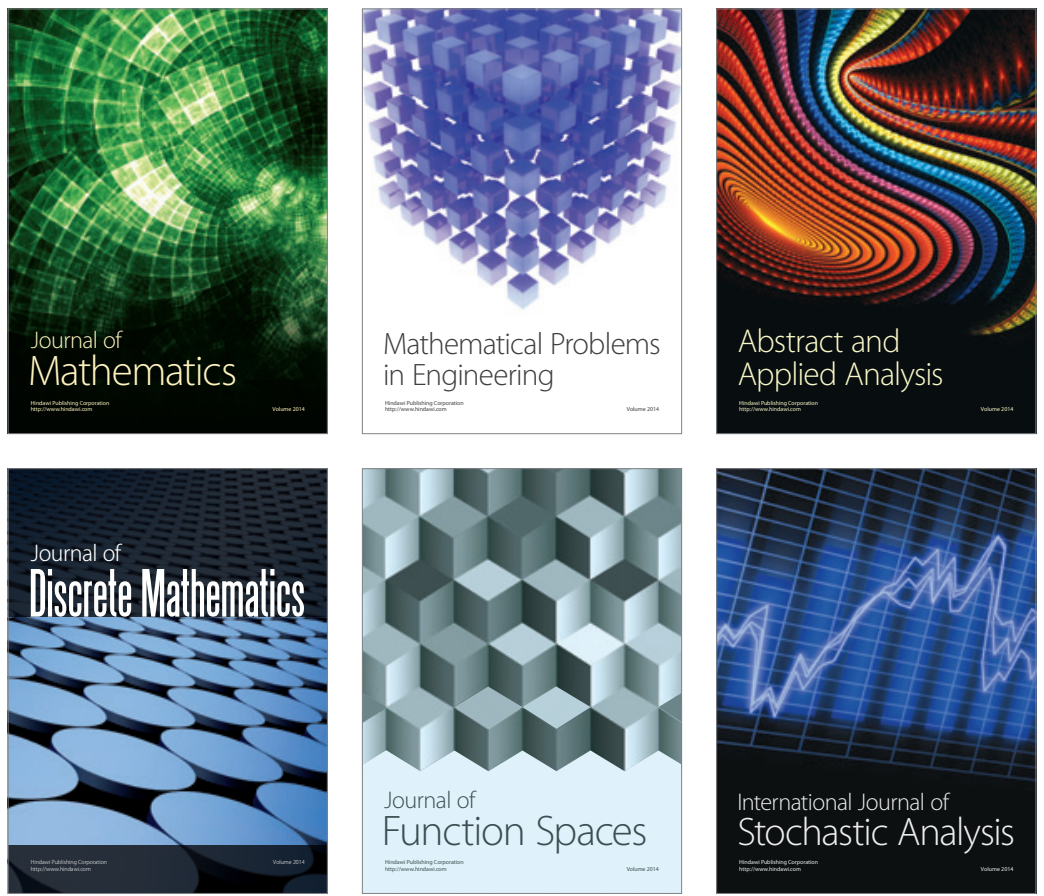

Journal of

Function Spaces

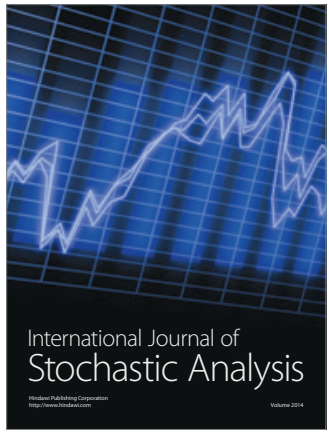

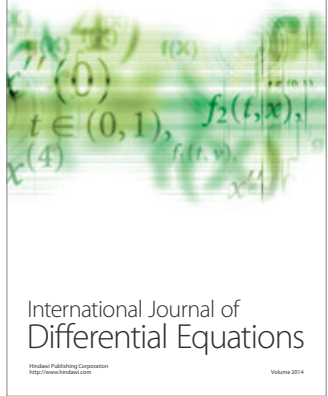
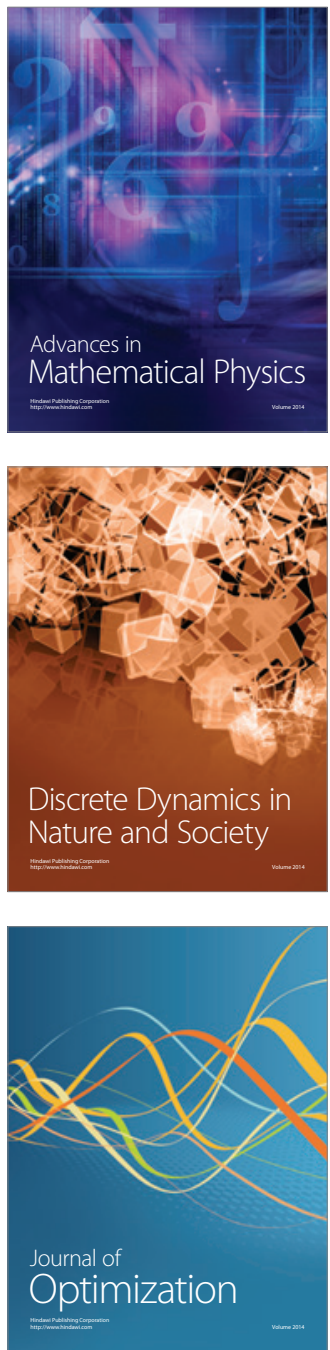